Manuscript for Review

\title{
Birefringent dispersive FDTD subgridding scheme
}

\begin{tabular}{|r|l|}
\hline Journal: & Electronics Letters \\
\hline Manuscript ID & ELL-2016-1709.R1 \\
\hline Manuscript Type: & Letter \\
\hline Date Submitted by the Author: & 13 -May-2016 \\
\hline Complete List of Authors: & $\begin{array}{l}\text { De Deckere, Bert; Ghent university, Information Technology } \\
\text { Van Londersele, Arne; Ghent university, Information Technology } \\
\text { De Zutter, Daniel; Ghent university, Information Technology } \\
\text { Vande Ginste, Dries; Ghent university, Information Technology }\end{array}$ \\
\hline Keywords: & $\begin{array}{l}\text { Hybrid Implicit Explicit (HIE), FINITE-DIFFERENCE TIME DOMAIN } \\
\text { ANALYSIS, BIREFRINGENCE, DISPERSION (WAVE), multiscale, subgridding }\end{array}$ \\
\hline &
\end{tabular}

SCHOLARONE

Manuscripts 


\section{Birefringent dispersive FDTD subgridding scheme}

Bert De Deckere, Arne Van Londersele, Daniël De Zutter, Dries Vande Ginste

A novel 2D FDTD subgridding method is proposed, only subject to the Courant limit of the coarse grid. By making $\mu$ or $\epsilon$ inside the subgrid dispersive, unconditional stability is induced at the cost of a sparse, implicit set of update equations. By only adding dispersion along preferential directions, it is possible to dramatically reduce the rank of the matrix equation that needs to be solved.

Introduction: The FDTD method was first introduced by Kane Yee in 1966 [1] and it allows the efficient computation of Maxwell's equations in the time domain. However, it suffers from two serious drawbacks. First, the maximum time step is limited by the smallest cell size, which makes it hard to model multiscale devices. Second, any straightforward refinement is obtained by squeezing the grid together, such that the refinement has a global influence along one (or more) of the main axes of the grid. Both of these problems can be tackled by introducing a subgrid where the update equations are solved implicitly. This allows for local refinement without the need to reduce the time step.

Many methods have been put forward to locally refine the grid. In essence, there are always two problems that need to be solved: a set of update equations needs to be defined in the refined region and a method has to be constructed to deal with the interface between the main grid and the subgrid. Using Huygens surfaces [2] for example, it is possible to transfer fields between the main grid and the (implicit) subgrid using equivalent currents. This method, however, suffers from late-time instability.

New implicit subgrid update equations in $x$ - and $y$-direction: The discussion below is restricted to the 2D TE-case. The whole simulation space, i.e. main grid and subgrid, is modeled by Yee cells (see Fig. 1). $H_{z}$ is discretized at integer space steps $i \Delta x, j \Delta y$ and half-integer time

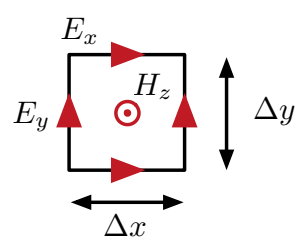

Fig. 1. Classical 2D Yee-cell for the TE mode.

steps $(n+1 / 2) \Delta t$, with the usual FDTD-notation $\left.H_{z}\right|_{i, j} ^{n+1 / 2}$. Similarly, the discretized electric fields are denoted $\left.E_{x}\right|_{i, j+1 / 2} ^{n},\left.E_{y}\right|_{i+1 / 2, j} ^{n}$. For conciseness, we first define the following difference operator

$$
\left.\mathcal{D}_{x} f\right|_{p, q} ^{m}=\frac{\left.f\right|_{p+1 / 2, q} ^{m}-\left.f\right|_{p-1 / 2, q} ^{m}}{\Delta x}
$$

i.e. the $x$-derivative of $f$ at $p \Delta x, q \Delta y$ and time instant $m \Delta t$. The analogous difference operators with respect to $y$ and $t$ are denoted $\mathcal{D}_{y}$ and $\mathcal{D}_{t}$. We also introduce the interpolation operator defined by

$$
\left.\mathcal{I}_{t} f\right|_{p, q} ^{n}=\frac{\left.f\right|_{p, q} ^{n+1}+\left.2 f\right|_{p, q} ^{n}+\left.f\right|_{p, q} ^{n-1}}{4}
$$

Using (1), the conventional FDTD update equations [1] can be written as

$$
\begin{aligned}
\left.\mathcal{D}_{t} H_{z}\right|_{i, j} ^{n} & =\left.\frac{1}{\mu} \mathcal{D}_{y} E_{x}\right|_{i, j} ^{n}-\left.\frac{1}{\mu} \mathcal{D}_{x} E_{y}\right|_{i, j} ^{n} \\
\left.\mathcal{D}_{t} E_{x}\right|_{i, j+1 / 2} ^{n+1 / 2} & =\left.\frac{1}{\epsilon} \mathcal{D}_{y} H_{z}\right|_{i, j+1 / 2} ^{n+1 / 2} \\
\left.\mathcal{D}_{t} E_{y}\right|_{i+1 / 2, j} ^{n+1 / 2} & =-\left.\frac{1}{\epsilon} \mathcal{D}_{x} H_{z}\right|_{i+1 / 2, j} ^{n+1 / 2},
\end{aligned}
$$

The set of equations (3)-(5) can be combined to yield the discrete version of the wave equation

$$
\left.\mu \in \mathcal{D}_{t}^{2} H_{z}\right|_{i, j} ^{n}=\left.\mathcal{D}_{x}^{2} H_{z}\right|_{i, j} ^{n}+\left.\mathcal{D}_{y}^{2} H_{z}\right|_{i, j} ^{n}
$$

with, e.g.,

$$
\left.\mathcal{D}_{x}^{2} H_{z}\right|_{i, j} ^{n}=\frac{\left.H_{z}\right|_{i+1, j}-\left.2 H_{z}\right|_{i, j} ^{n}+\left.H_{z}\right|_{i-1, j} ^{n}}{\Delta x^{2}} .
$$

The reader can check that $\mathcal{D}_{x}^{2}$ indeed corresponds to the repeated application of $\mathcal{D}_{x}$, i.e. $\mathcal{D}_{x}^{2} f=\mathcal{D}_{x}\left(\mathcal{D}_{x} f\right)$. As in [3], we can define the extended $Z$-transform of $H_{z}$, with $U, V$ and $Z$ the transform variables corresponding to $x, y$ and $t$.

$$
\hat{H}_{z}(U, V, Z)=\left.\sum_{i=-\infty}^{\infty} \sum_{j=-\infty}^{\infty} \sum_{n=-\infty}^{\infty} H_{z}\right|_{i, j} ^{n} Z^{n} U^{i} V^{j}
$$

Applying this transformation to (6) yields

$$
\mu \epsilon \frac{(Z-1)^{2}}{Z \Delta t^{2}} \hat{H}_{z}=\frac{(U-1)^{2}}{U \Delta x^{2}} \hat{H}_{z}+\frac{(V-1)^{2}}{V \Delta y^{2}} \hat{H}_{z} .
$$

The response for a plane-wave is obtained by setting [3]

$$
Z=e^{j \omega \Delta t}, \quad U=e^{j k_{x} \Delta x}, \quad V=e^{j k_{y} \Delta y},
$$

such that we arrive at the well-known dispersion relation

$$
\mu \epsilon \sin ^{2}\left(\frac{\omega \Delta t}{2}\right)=\frac{\Delta t^{2}}{\Delta x^{2}} \sin ^{2}\left(\frac{k_{x} \Delta x}{2}\right)+\frac{\Delta t^{2}}{\Delta y^{2}} \sin ^{2}\left(\frac{k_{y} \Delta y}{2}\right) .
$$

The reason for instability is that the right-hand side can assume values larger than $\mu \epsilon$, whereas the left-hand side cannot. This leads to the traditional Courant limit

$$
\Delta t<\sqrt{\frac{\mu \epsilon}{\frac{1}{\Delta y^{2}}+\frac{1}{\Delta x^{2}}}} .
$$

This limit can therefore be traced back to the fact that the $\sin ^{2}$-function in the left-hand side of (11) is bounded by unity. To mitigate this, we can make either $\epsilon$ or $\mu$ dispersive. Let us first modify $\epsilon$ by introducing the following substitution in the $Z$-domain.

$$
\frac{1}{\epsilon} \rightarrow \frac{1}{\epsilon} \frac{(Z+1)^{2}}{4 Z}
$$

The action of this operator on a function $f$ translates to the time domain as

$$
\frac{1}{\epsilon} \frac{(Z+1)^{2}}{4 Z} \hat{f} \rightarrow \frac{1}{\epsilon} \mathcal{I}_{t} f
$$

Applying this to (3)-(5) yields

$$
\begin{aligned}
\left.\mathcal{D}_{t} H_{z}\right|_{i, j} ^{n} & =\left.\frac{1}{\mu} \mathcal{D}_{y} E_{x}\right|_{i, j} ^{n}-\left.\frac{1}{\mu} \mathcal{D}_{x} E_{y}\right|_{i, j} ^{n} \\
\left.\mathcal{D}_{t} E_{x}\right|_{i, j+1 / 2} ^{n+1 / 2} & =\left.\frac{1}{\epsilon} \mathcal{I}_{t} \mathcal{D}_{y} H_{z}\right|_{i, j+1 / 2} ^{n+1 / 2} \\
\left.\mathcal{D}_{t} E_{y}\right|_{i+1 / 2, j} ^{n+1 / 2} & =-\left.\frac{1}{\epsilon} \mathcal{I}_{t} \mathcal{D}_{x} H_{z}\right|_{i+1 / 2, j} ^{n+1 / 2} .
\end{aligned}
$$

The corresponding dispersion relation (13) now takes the form

$$
\mu \epsilon \tan ^{2}\left(\frac{\omega \Delta t}{2}\right)=\frac{\Delta t^{2}}{\Delta x^{2}} \sin ^{2}\left(\frac{k_{x} \Delta x}{2}\right)+\frac{\Delta t^{2}}{\Delta y^{2}} \sin ^{2}\left(\frac{k_{y} \Delta y}{2}\right) .
$$

As the $\sin ^{2}$ function in the 1.h.s. is replaced by $\tan ^{2}$, this readily implies that the update scheme (15)-(17) is unconditionally stable, but no longer explicit. Note that this dispersion relation is the same as for the unconditionally stable Crank-Nicholson scheme [4]. Further note that (15) does not differ from (3). This is crucial as this will later allow us to use $E_{x}$ and $E_{y}$ in the classical Yee grid and glue it together with $H_{z}$ of the subgrid in a stable way. An alternative set of update equations is obtained by making $\mu$ dispersive in a similar way to $\epsilon$.

Birefringent subgridding: Above, the subgridding simultaneously affects the $x$ - and $y$-direction. Let us now switch to a birefringent (or biaxial) medium with the following dielectric tensor, while leaving $\mu$ unchanged

$$
\overline{\bar{\epsilon}}=\epsilon_{0} \operatorname{diag}\left(\epsilon_{x x}, \epsilon_{y y}, \epsilon_{z z}\right)
$$

Suppose we are only interested in applying the subgridding in the $x$-direction (using an implicit FDTD-scheme) but want to leave the 
$y$-direction unchanged, i.e. explicit. To this end the following substitution is invoked

$$
\frac{1}{\epsilon_{y y}} \rightarrow \frac{1}{\epsilon_{y y}} \frac{(Z+1)^{2}}{4 Z} .
$$

The FDTD update equations (3)-(5) are now transformed to

$$
\begin{aligned}
& \left.\mathcal{D}_{t} E_{x}\right|_{i, j+1 / 2} ^{n}=\left.\frac{1}{\epsilon_{0} \epsilon_{x x}} \mathcal{D}_{y} H_{z}\right|_{i, j+1 / 2} ^{n} \\
& \left.\mathcal{D}_{t} E_{y}\right|_{i+1 / 2, j} ^{n}=-\left.\frac{1}{\epsilon_{0} \epsilon_{y y}} \mathcal{I}_{t} \mathcal{D}_{x} H_{z}\right|_{i+1 / 2, j} ^{n} \\
& \left.\mathcal{D}_{t} H_{z}\right|_{i, j} ^{n+1 / 2}=\left.\frac{1}{\mu} \mathcal{D}_{y} E_{x}\right|_{i, j} ^{n+1 / 2}-\left.\frac{1}{\mu} \mathcal{D}_{x} E_{y}\right|_{i, j} ^{n+1 / 2} .
\end{aligned}
$$

When setting $\epsilon_{x x}=\epsilon_{y y}=\epsilon_{r}$ and $\mu=\mu_{0} \mu_{r}$ the dispersion relation corresponding to (21)-(23) is readily found to be

$$
\frac{\epsilon_{r} \mu_{r}}{(c \Delta t)^{2}}=\frac{1}{\Delta x^{2}} \frac{\sin ^{2}\left(\frac{k_{x} \Delta x}{2}\right)}{\tan ^{2}\left(\frac{\omega \Delta t}{2}\right)}+\frac{1}{\Delta y^{2}} \frac{\sin ^{2}\left(\frac{k_{y} \Delta y}{2}\right)}{\sin ^{2}\left(\frac{\omega \Delta t}{2}\right)},
$$

which indeed shows unconditional stability along $x$. Based on all of the above, it can in general be stated that the transformation

$$
\mathcal{D}_{\alpha} \rightarrow \mathcal{I}_{t} \mathcal{D}_{\alpha} \quad(\alpha=x \text { or } y)
$$

in either the $E$ or $H$ update equations, yields unconditional stability along the spatial dimension $\alpha$. For clarity, the losses have been neglected up until now. These can however easily be incorporated. The update equations for a lossy medium are

$$
\begin{aligned}
\left.\mathcal{D}_{t} H_{z}\right|_{i, j} ^{n} & =\left.\frac{1}{\mu} \mathcal{D}_{y} E_{x}\right|_{i, j} ^{n}-\left.\frac{1}{\mu} \mathcal{D}_{x} E_{y}\right|_{i, j} ^{n} \\
\left.E_{x}\right|_{i, j+1 / 2} ^{n+1} & =\left.C_{1} E_{x}\right|_{i, j+1 / 2} ^{n}+\left.C_{2} \mathcal{D}_{y} H_{z}\right|_{i, j+1 / 2} ^{n+1 / 2} \\
\left.E_{y}\right|_{i+1 / 2, j} ^{n+1} & =\left.C_{1} E_{y}\right|_{i+1 / 2, j} ^{n}-\left.C_{2} \mathcal{D}_{x} H_{z}\right|_{i+1 / 2, j} ^{n+1 / 2} \\
C_{1} & =\frac{2 \epsilon-\sigma \Delta t}{2 \epsilon+\sigma \Delta t}, \quad C_{2}=\frac{2 \Delta t}{2 \epsilon+\sigma \Delta t} .
\end{aligned}
$$

The substitution (25) remains applicable for lossy media.

Interface main grid and subgrid: Using the interface depicted on Fig. 2, it is possible to obtain a provably stable asymmetric refinement of the grid. The fields are denoted with uppercase letters in the main grid and lowercase letters in the subgrid. If a field component from the subgrid $\left.h_{z}\right|_{i, j}$ is used for the update of $\left.E_{x}\right|_{i, j+1 / 2}$ from the main grid, or similarly in the reverse direction, then a weight factor $w$ is defined as

$$
\left.E_{x}\right|_{i, j+1 / 2} ^{n+1}=\left.E_{x}\right|_{i, j+1 / 2} ^{n}+\frac{\Delta t}{\epsilon} \frac{\left.H_{z}\right|_{i, j+1} ^{n+1 / 2}-\left.w \sum_{m} h_{z}^{(m)}\right|_{i, j} ^{n+1 / 2}}{\Delta y_{j+1 / 2}} .
$$

These weight factors depend on the refinement ratio, as illustrated in Fig. 2.

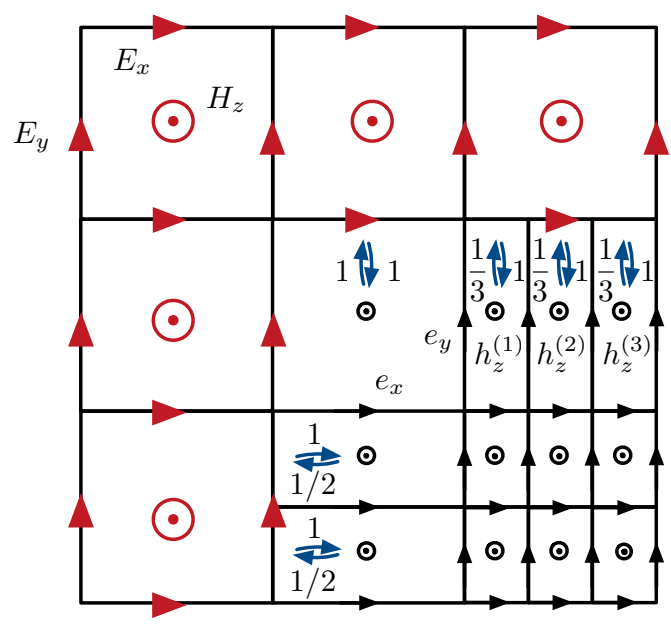

Fig. 2 Part of an interface between the main grid and the subgrid with asymmetric refinement ratio of $3 \times 2$. The weights next to the blue arrows are the weight factors $w$ defined in (30).
Accuracy: The set of update equations remain second-order accurate in time and space in the bulk of the subgrid, just as is the case for the regular FDTD update equations.

Transmission through a conductive slab: To illustrate the accuracy, we investigate the transmission through a conductive slab $(\sigma=500 \mathrm{~S} / \mathrm{m})$ with thickness $d=3 \mathrm{~mm}$ and height $2 \mathrm{~m}$, illuminated by a broadband $z$-oriented magnetic line source at $x_{0}=-0.1 \mathrm{~m}$. At the backside of the conductive slab, is a sensor at height $h$, which monitors the $H_{z}$ field. In the main grid, $\Delta x=\Delta y=5 \mathrm{~mm}$. The slab is embedded at the center of a birefringent subgrid with width $15 \mathrm{~mm}$ and height $1.98 \mathrm{~m}$. The height is chosen such that edge diffraction effects at the location of the sensor can be neglected. The refinement ratio in the $x$-direction is 100, i.e. $w=0.01$. The equations governing the subgrid are (26)-(28) with $\mathcal{D}_{x} \rightarrow \mathcal{I}_{t} \mathcal{D}_{x}$ in (28). The results are presented in Fig. 4 for two values of $h$ and, compared to an analytical solution, demonstrate an excellent accuracy.

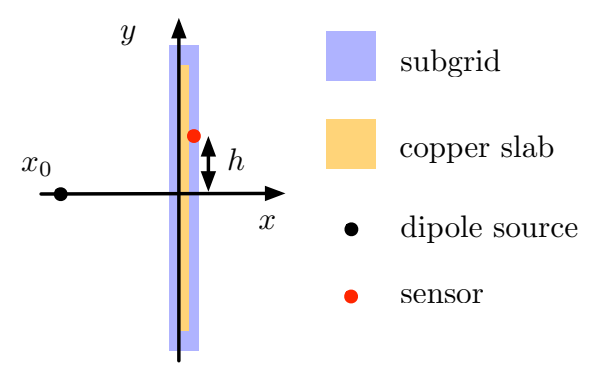

Fig. 3 Setup to measure the transmission through a conductive slab illuminated by a dipole source.

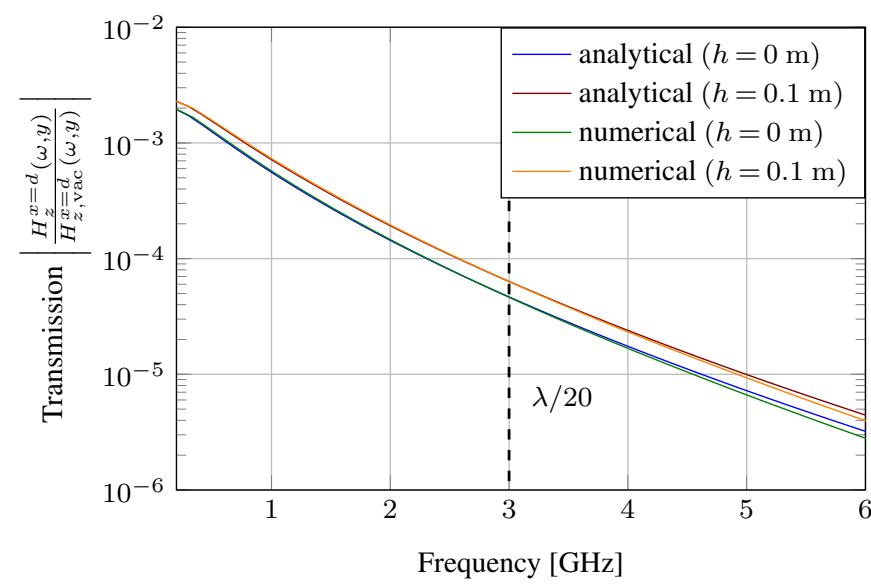

Fig. 4 Ratio of field amplitudes with and without conductive slab. At $3 \mathrm{GHz}$ the course grid sampling is $\lambda / 20$.

Conclusion: A novel implicit, unconditionally stable FDTD subgridding method was proposed and numerically validated. Although not discussed here, this method is provably stable. Further, the proposed method allows for refinement along preferential directions to reduce the rank of the sparse matrix involved.

Bert De Deckere, Arne Van Londersele, Daniël De Zutter, Dries Vande Ginste (Dept. of Information Technology, Ghent University, Belgium)

E-mail: bert.dedeckere@icloud.com

\section{References}

1 Yee, K. S.: 'Numerical solution of initial boundary value problems involving Maxwell's equations in isotropic media', IEEE Transactions on Antennas and Propagation, 1966, 14, pp. 302-307

2 Bérénger, J. P.: 'A Huygens subgridding for the FDTD method', IEEE Transactions on Antennas and Propagation, 2006, 54, pp. 3797-3804

3 Weedon, W. H. and Rappaport, C. M.:'A General Method for FDTD Modeling of Wave Propagation in Arbitrary Frequency-Dispersive Media', IEEE Transactions on Antennas and Propagation, 1997, 45, pp. 401-410

4 Sun, G. and Trueman C. W.: 'Unconditionally stable Crank-Nicolson scheme for solving two-dimensional MaxwellâĂŹs equations', Electronics Letters, 2003, 39, pp. 595-597 